\title{
Analyzing Interaction for Automated Adaptation - First Steps in the IAAA Project
}

\author{
Thomas Neumayr, Daniel Kern, Mirjam Augstein, Werner Kurschl, and Josef Altmann
}

\begin{abstract}
Because of an aging society and the relevance of computer-based systems in a variety of fields of our life, personalization of software systems is becoming more important by the day in order to prevent usage errors and create a good user experience. However, personalization typically is a timeconsuming and costly process if it is done through manual configuration. Automated adaptation to specific users' needs is, therefore, a useful way to reduce the efforts necessary. The IAAA project focuses on the analysis of user interaction capabilities and the implementation of automated adaptations based on them. However, the success of these endeavors is strongly reliant on a careful selection of interaction modalities as well as profound knowledge of the target group's general interaction behavior. Therefore, as a first step in the project, an extensive task-based user observation with thorough involvement of the actual target group was conducted in order to determine input devices and modalities that would in a second step become subject of the first prototypic implementations. This paper discusses the general objectives of the IAAA project, describes the methodology and aims behind the user observation and presents its results.
\end{abstract}

Keywords-personalization, human-computer interaction, adaptation, inclusion

\section{INTRODUCTION}

A $\mathrm{N}$ ever expanding role of personalization in computerbased systems can be expected due to increased and changing requirements for these systems caused by rapid and lasting demo-graphic change, prospectively aging employees or the focus on intelligent production processes, which are all topics dealt with through the European and national Strategy 2020. Further-more, the portion of people with disabilities in the workforce can be increased through personalized hard- and software. However, the personalized configuration of interactive systems implies an enormous amount of time and effort if it is not done automatically respectively semiautomatically. Consequently, the demand for interactive systems which can automatically adapt to special needs of individual types of users will rise.

People with disabilities were chosen as the initial target group because the diversity of their needs is best suited for personalization. However, the Interaction Analysis for Automated Adaptation (IAAA) project does not exclusively

T. Neumayr and D. K. are with Research \& Development, UAS Upper Austria, Hagenberg, Austria (e-mail: thomas.neumayr@fh-hagenberg.at; daniel.kern@fh-hagenberg.at)

M. Augstein and J. Altmann are with Communication and Knowledge Media, UAS Upper Austria, Hagenberg, Austria (e-mail: mirjam.augstein@fhhagenberg.at, josef.altmann@fh-hagenberg.at

W. Kurschl is with Human-Centered Computing, UAS Upper Austria, Hagenberg, Austria ( e-mail: werner.kurschl@fh-hagenberg.at). deal with the personalization of interactive systems, but also analyzes and provides means to choose among different input modalities. Therefore, an analysis tool capable of automated and systematic analyses of input behavior is developed. The tool's output can in a next step become the basis for making the chosen software adaptive (reducing configuration complexity).

\section{RELATED WORK}

In our early research, we identified two categories of interaction modalities which seemed promising for our target group: smartwatch combination with touchless or touch interaction as well as applying physical pressure.

Chen et al. showed that a combined usage of a smartphone and a smartwatch extends the interaction possibilities and thus enables a wider range of executable gestures, which is also relevant for people with disabilities [1].

As people with disabilities often have problems executing a gesture in the right way or hitting a small button on a smartphone, physical pressure can be used instead because it is more a question of gross motor skills than other input paradigms. There are many alternative methods to measure the physical pressure with a common smartphone.

Vibration absorption is one way of measuring the physical pressure. According to [2] it is possible to estimate the physical pressure by analyzing the changes of the accelerometer data while a vibration motor vibrates.

Another way to estimate the physical pressure of an interaction is to analyze the magnetic field around the smartphone. The approach which is described in [3] uses several pens with different magnetic attributes to determine the physical pressure on the pen.

An acoustic based approach of determining the physical pressure is described in [4]. In this way the smartphone plays a high-frequent sound through the built-in speakers and analyzes the feedback received through the microphone. The user applies pressure by covering the microphone with an object or finger.

The following paragraphs describe Fig. 1.

To find out which forms of interaction are potentially beneficial for people with disabilities, we designed some interaction scenarios first (1.), e.g., combination of a smartwatch with a touchless interaction device. Therefore we created some test cases whereas some of them were supported by small applications to evaluate motor and cognitive abilities (2.) (for a description of these tests see Section V). 

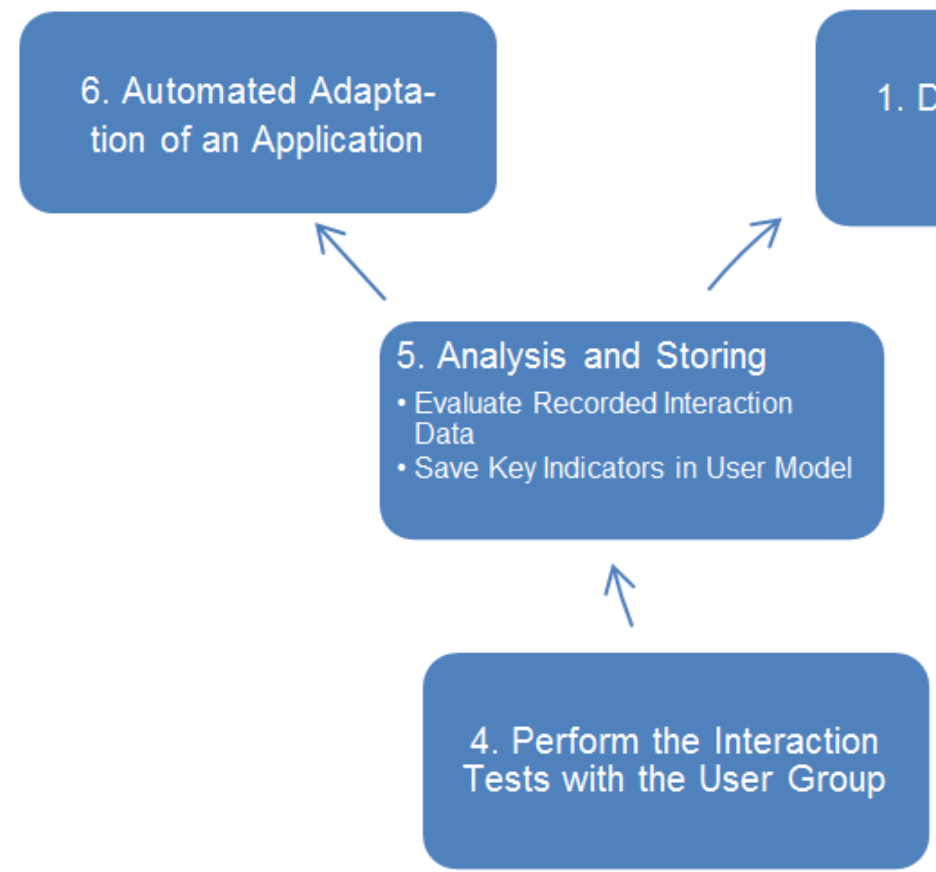

1. Design an Interaction Scenario

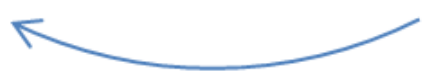

Fig. 1. Process diagram of IAAA's interaction analysis and adaption.

After having identified a set of interaction scenarios relevant to the target group, e.g., i) gesture-based, ii) haptic or iii) traditional (e.g., via mouse and keyboard) interaction and combinations of these, we developed several prototypes which aim at measuring users' interaction abilities regarding different aspects. The measurement of the user's interaction is based on some key indicators.

\section{MEthodOLOGY}

For instance, an abstract construct of accuracy could be introduced and calculated through a number of indicators like, e.g., i) the deviation between an executed gesture and the given reference gesture (see [5]), ii), the deviation of intended touchpoints to the target points on a touch screen (see [6]), or iii) the number of wrong key presses on a keyboard. To test the abilities of the users we developed some interaction tests for each interaction scenario (3.). One interaction test affects several key indicators. With the definition of a number of key indicators that cover the whole interaction, e.g., reaction speed, or time to achieve a task, the ever increasing possibility to predict if a user will be able to achieve his or her interaction goals using a particular input device arises.

The second test with the user group (4.) aims to analyze the execution of the interaction tests by the user group. In this step all key indicators based on the interaction scenario are computed and stored in the user model (5.). The user model which contains a comprehensive number of indicators should predict a user's ability to interact with a system, as outlined in [7].
In general this user model will comprise information about different kinds of input devices and related configurations. In addition to users' ability to operate a particular device properly, the user model should also account for the users' prospective individual preferences as they are likely to influence motivation and therefore the overall performance.

Finally the user model becomes the basis for the automated adaptation of further applications (6.).

\section{OBSERVATION}

This section describes a user observation we conducted in order to determine general insight into our first target group's interaction capabilities.

\section{A. Aims and Participants}

By conducting an early observation of the first target group, we aimed at gaining initial insights into participants' capability regarding motor abilities, devices currently used, as well as the type of software used predominantly. In early November 2014, we had the possibility to work with four participants at an IT workshop for people with disabilities in Hagenberg, Upper Austria, three of which were male and one female. We chose these four participants mainly because they have a variety of different diagnoses ranging from cognitive through motor (e.g. spastic paraplegia) to visual limitations. Furthermore their limitations are mostly not affecting all areas, e.g., one area (e.g. motor skills) may be strongly limited, whereas other areas (e.g. cognitive skills) might be on a similar level for nonimpaired persons. This aspect helps us isolate and discriminate among different types of motor movements and make connections to the type of impairment that might inhibit the chances of individuals being able to perform those movements (in a comfortable way). 


\section{B. Tasks/Test Cases}

We chose a task-based approach helping us to find out how well individual participants were able to work with several input modalities. For instance, we asked participants to touch all corners of a box that was open on one side (representing the detectable area of touch-less systems like Leap Motion1) to touch their wrist watch with a finger of the other hand to simulate smart watch interaction, and many more. While participants carried out the tasks, three observers took notes in an observation guide that was created in preparation of the observation. The guide contained some general information about participants and seven different functional areas which can be derived from the findings described in Section II:

(1) smart watch as output device of haptic feedback

(2) smart watch as source of additional sensor data (implicit)

(3) smart watch as additional explicit input device

(4) physical pressure

(5) magnetic field manipulation

(6) special touch gestures

(7) touch-less gestures

A total of approximately 50 tasks were authored mainly to measure participants' capabilities and their ease of use during the aforementioned fields. However, not all tasks were performed with all participants as some tasks were ruled out beforehand during the observations if it was clear that the current participant would not be able to perform them. Exemplarily, if it was not possible to reach one hand with the other, it would have been strongly discouraging to ask the participant to perform tasks requiring just this behavior. In the next section, we will describe the obtained results in more detail.

In addition to the observation itself, in some instances usage data has been directly recorded through prototypes on a smart phone, predominantly physical pressure, touch precision, and the amount of time a person was able to fulfill a touch without interruption. There has been a total of four runs (one with each participant) each lasting between 20 and 30 minutes.

\section{RESULTS}

The results section presents figures that depict the arithmetic mean of the three observers' rating/evaluation of how well participants were able to perform a specific task.

\section{A. Smart watch}

1) Smart watch as output device of haptic feedback

It has been observed in prior research that in the domain of people with disabilities it is a somewhat common scenario that users unintendedly leave the area recognizable by a common touch-less motion recognizing input device like Leap Motion without noticing it (see [5]). The application of a smart watch on one arm (or even on both arms) could therefore be used to signal the leaving to the user by vibrating or playing a sound, etc. To get some initial data about the applicability of this approach, we asked participants to move their hand (with fingers extended or closed, as they wished) to every side of a box that was open on one side and dimensions identical to those of the Leap Motion's detectable area.

With a few exceptions participants managed to reach all sides of the box (see Fig. 2).

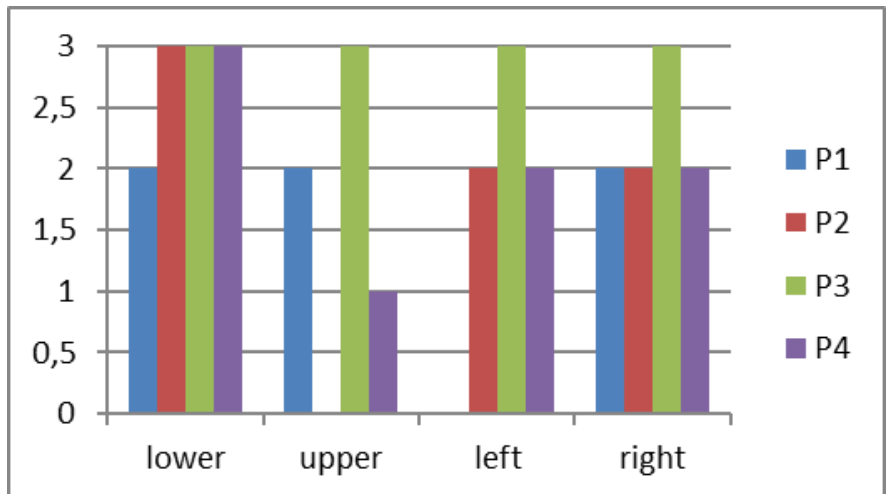

Fig. 2. Participants' (P1-P4) ability to reach sides of a box. 3 means reached well, 2 average, 1 badly, 0 wasn't reached at all.

2) Smart watch as source of additional sensor data (implicit) In this category, we exemplarily asked participants to hold a smartphone in their hand, reverse it to its back side and then touch the screen with the thumb (of the hand holding the device) or to simply extend their fingers and touch the desk they were sitting in front of with the back side of their fingers (see Fig. 3). We in that way intended to find out if rotation movements of arms were sufficiently possible for the target group. Other results in that field are depicted in Fig. 4, Fig. 5, and Fig. 6. Please refer to the captions which describe the tasks in more detail. The results show that a wide area of different interactions are possible. However, some of them are highly dependent on the individual inhibitions participants suffered from which mainly becomes visible in the task that is depicted in Fig. 6 where two participants simply were not able to rotate their hand $180^{\circ}$ and therefore could not fulfill the task. Yet, the other two participants did not experience any problems.

\section{3) Smart watch as additional explicit input device}

Here, participants should touch their wrist watch with a finger of the other hand and hold the touch for several seconds (we

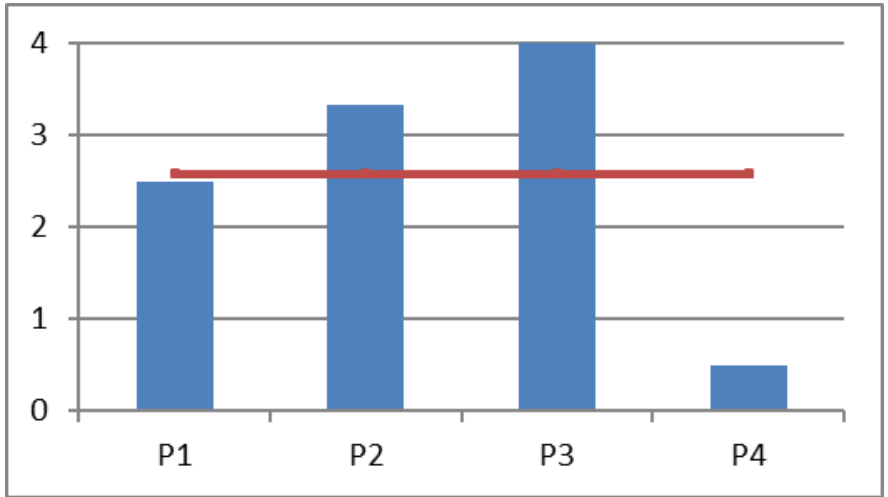

Fig. 3. Participants' ability to turn their hand around holding a smartphone and then executing touch. 4 means "worked very well” whereas 0 means "did not work at all”.

\footnotetext{
${ }^{1}$ https://www.leapmotion.com/ last accessed May $28^{\text {th }} 2015$
} 


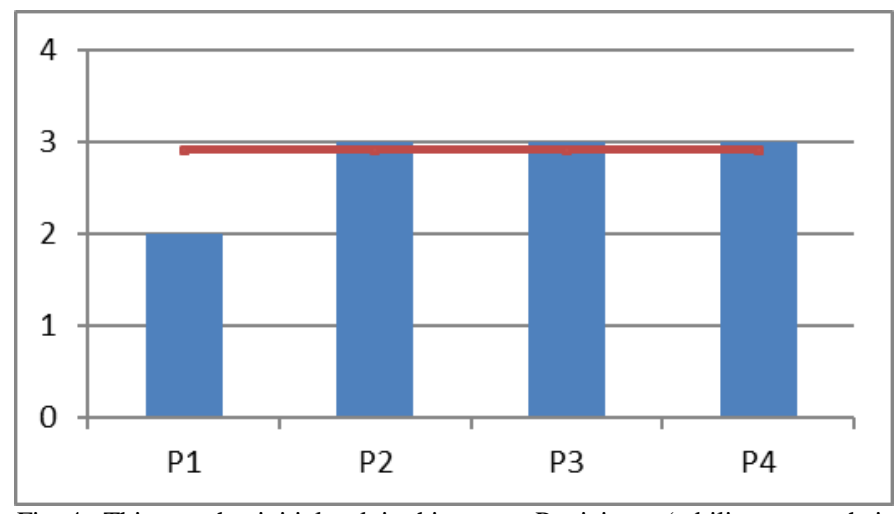

Fig. 4. This was the initial task in this group. Participants` ability to turn their hand to its backside and then to the initial position, afterwards performing a touch onto a table surface. 4 means "worked very well” whereas 0 means "did not work at all”.

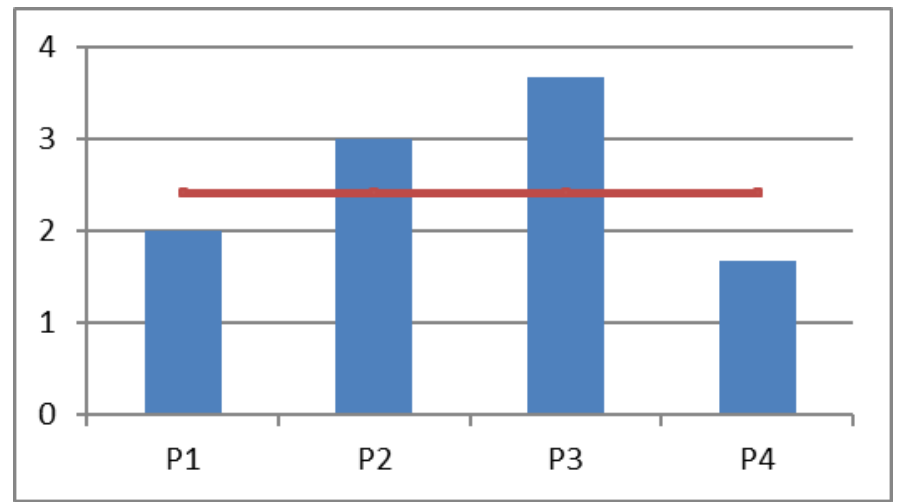

Fig. 5. The task here was to extend a finger, turn the hand $90^{\circ}$ and lay the finger on the table. 4 means "worked very well" whereas 0 means "did not work at all”.

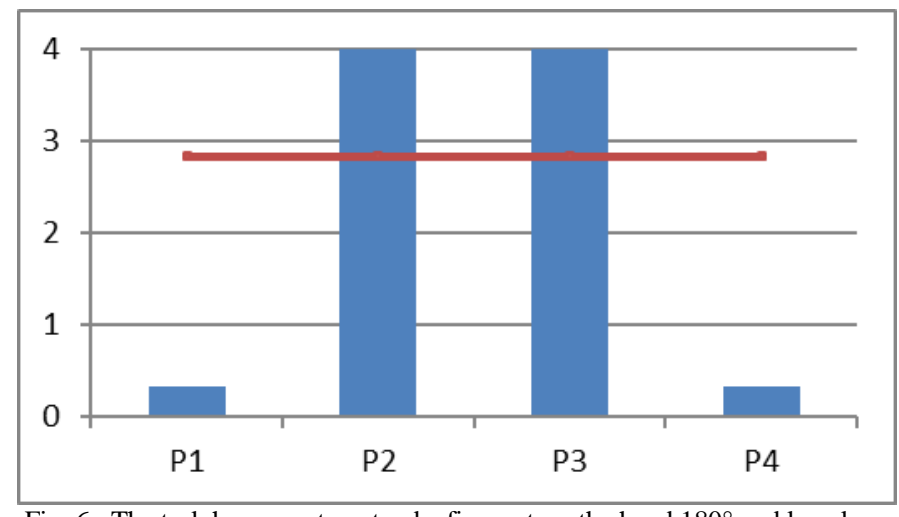

Fig. 6. The task here was to extend a finger, turn the hand $180^{\circ}$ and knock on the table. 4 means "worked very well” whereas 0 means "did not work at all”.

see Fig. 7. We decided to omit this task with participant P1 for reasons described in Section IV.B.

\section{A. Physical Pressure}

Participants were asked to apply pressure onto a smartphone with their hand and consequently vary the amount of pressure applied (see Fig. 8). The smartphone could either rest on a table and be pressed as a whole onto the table top or be held in hand while applying pressure. During this task, some usage data has been recorded with a simple prototype application, which will be presented in a future publication.
T. NEUMAYR, D. KERN, M. AUGSTEIN, W. KURSCHL, J. ALTMANN

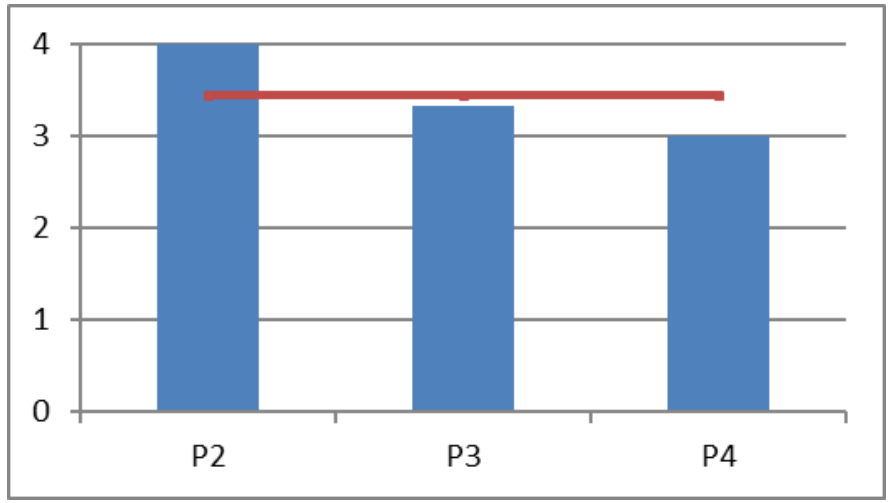

(a)

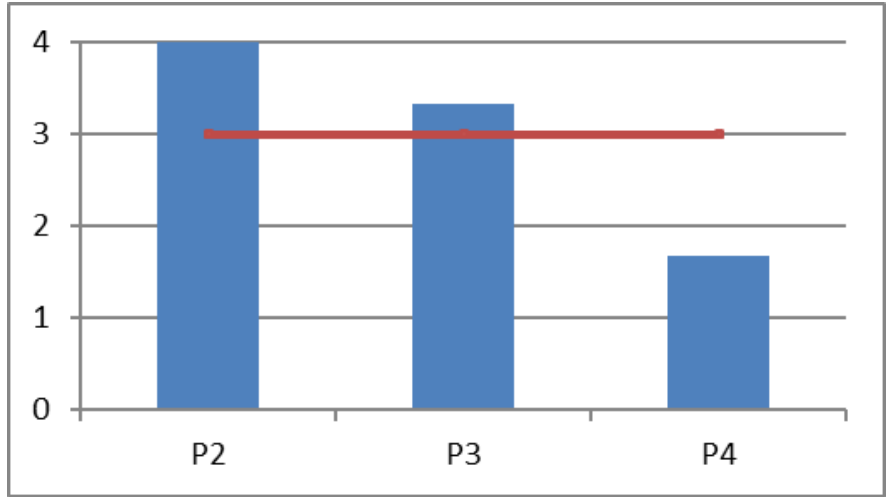

(b)

Fig. 7. Participants' capabilities touching a wrist watch with a finger of the other hand (a) and hold it steadily for several seconds (b).

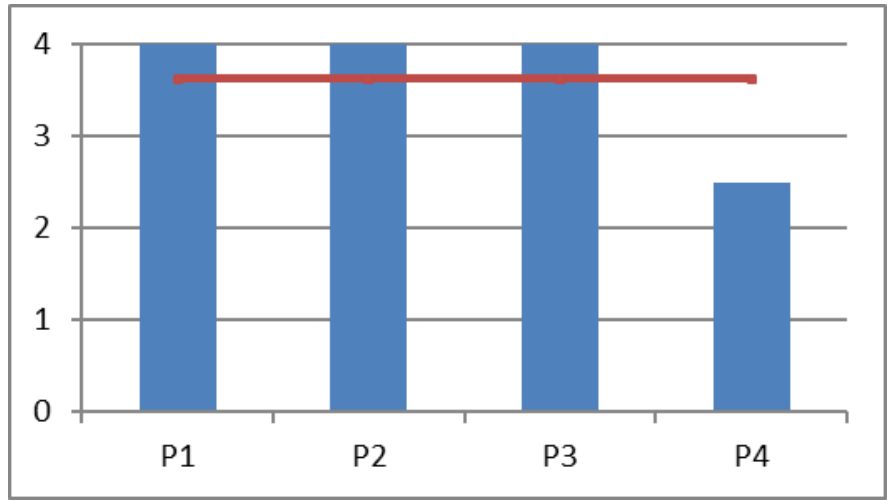

Fig. 8. Observation results of participants applying pressure to a smartwatch display and varying the pressure.

\section{B. Magnetic Field Manipulation}

For this task participants were asked to hold a stylus pen for some period of time, rotate the pen in both directions while it was lying on a table and afterwards draw specific figures on an empty sheet of paper, e.g., circles, rectangles, and swipes in four different directions. Every participant was fully able to hold the pen but most had difficulties drawing recognizable figures on the sheets (see Fig. 9). Additionally, we experimented with a Rubik's Cube and a Tennis ball to see how well participants could handle such objects. Of course, these objects could later be prepared to contain magnets and 
then be used for interaction. However, as can be seen in

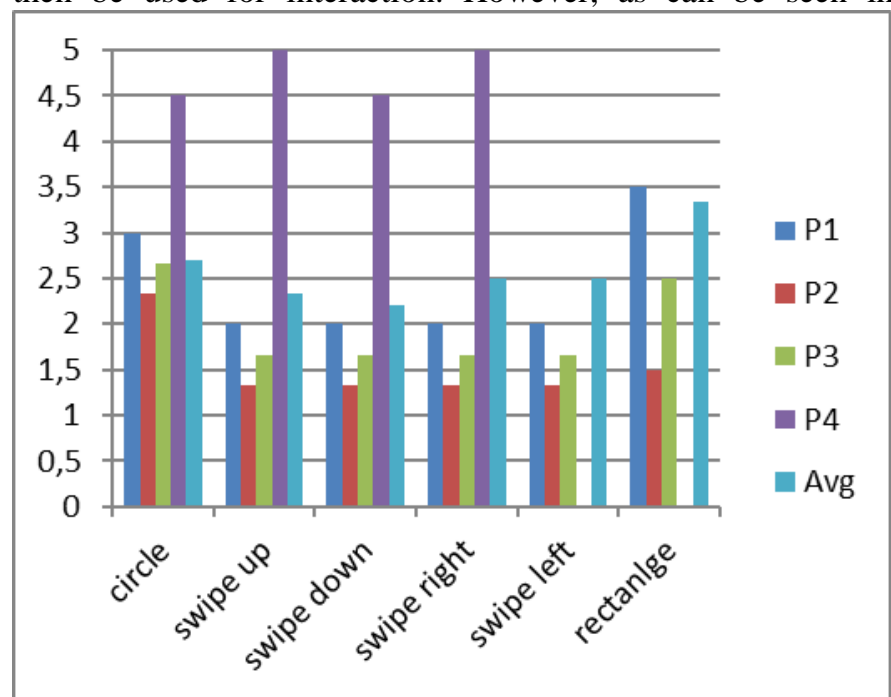

Fig. 9. Participants’ capabilities of drawing different figures on a sheet.

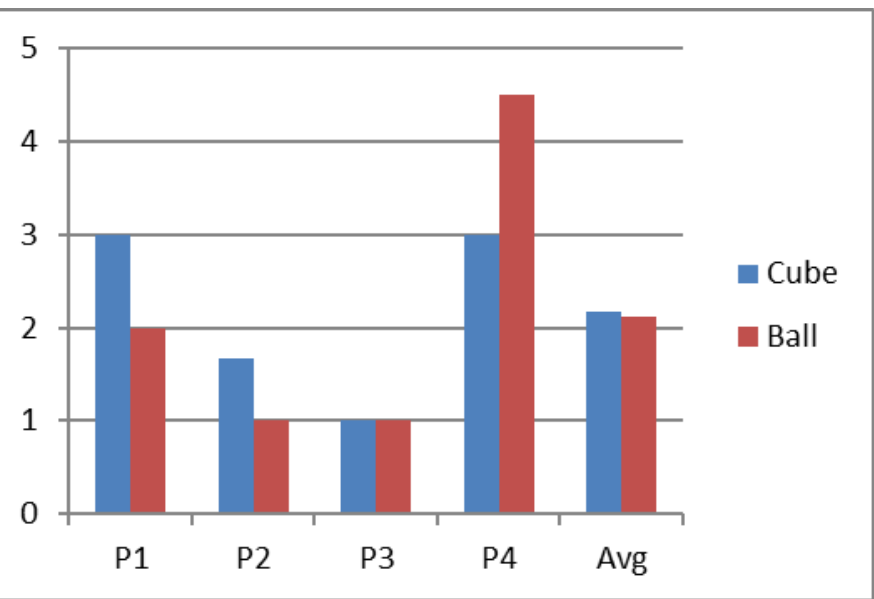

Fig. 10. Participants’ ability to control and move a Rubik’s Cube and a Tenni ball (which could later be used for interaction purposes). 5 means that it worked very well, 0 means that it did not work at all.

Fig. 10, both the ball and the cube did not suffice in providing a reliable means to interact. The cube could hardly be turned over by many participants whereas the Tennis ball left the area of reach unintendedly in many occasions.

\section{Special Touch Gestures}

For this task some sheets of paper were prepared beforehand by the research team that showed shapes participants should use as templates to hold or grab fictitious objects like a sponge, a pen or a computer mouse (as described in [8]). However, the participants in general were not able or could only manage with a lot of time and effort to simulate holding such objects in a way that would allow for the reliable and automated recognition of the gestures' touch points. Therefore this approach was not regarded as a good starting point following the observation.

\section{Touch-Less Gestures}

Parts of the related interaction capabilities have already been analyzed with the tasks described in Section A.1) where we intended to find out if participants were capable of making use of the recognizable three-dimensional area typical to touch-less

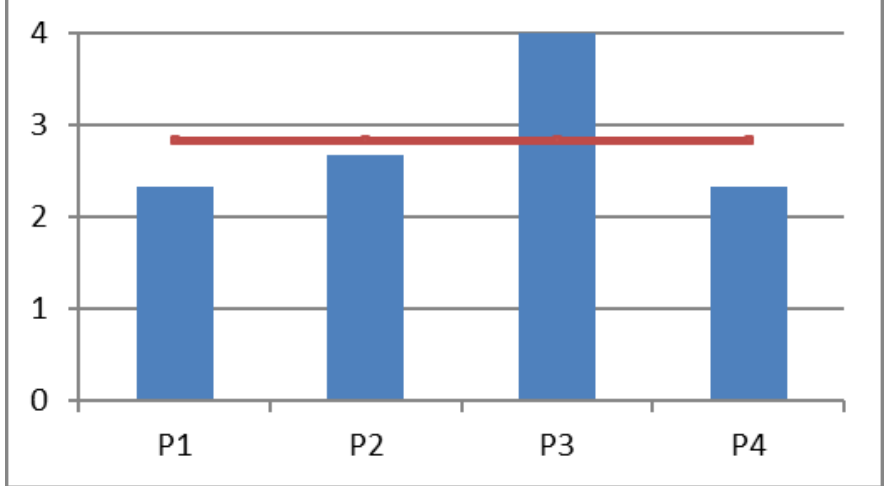

Fig. 11. Participants' (P1-P4) ability to reach all regions of a detectable area in the approximate size of a Leap Motion controller.

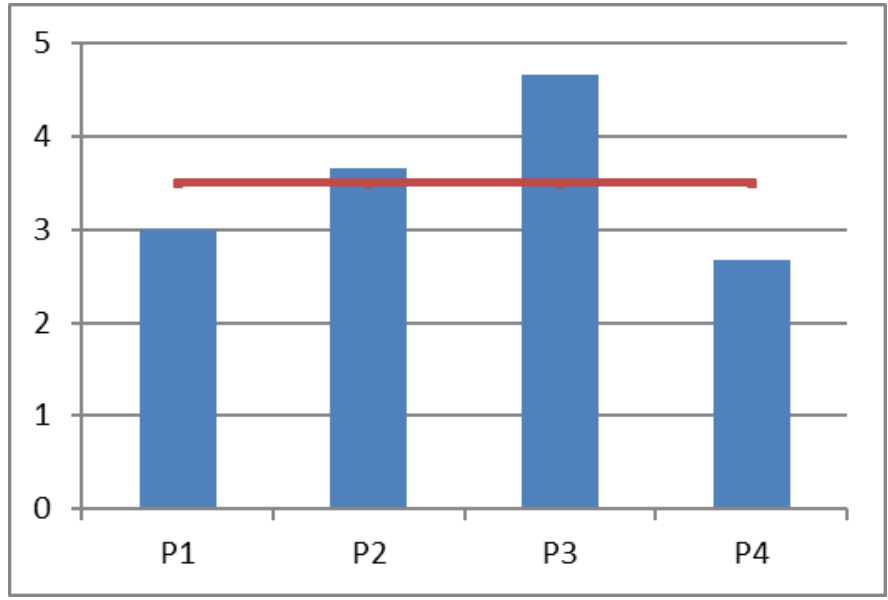

Fig. 12. How big was the region that could be sufficiently reached by the participants (P1-P4)? 5 represents a large region, comparable to reaching all areas.

input devices like Leap Motion. A more general depiction of the interaction capabilities for touchless-interaction in general can be seen in Fig. 11 and Fig. 12. Additionally, we aimed at discovering if interaction by extending a differing number of fingers at a time was possible (see Fig. 13 for the results). The results do not seem very promising for some participants as we observed that the singular extension of fingers required a lot of effort for most of them, whereas participant one was only able to extend all fingers (of the right hand) at once and not successively.

\section{Discussion}

In this paper we presented the results of an initial observation with our first target group, namely people with disabilities. The results were intended to guide the research team into directions of input modalities that are generally usable by the target group regarding their motor and cognitive capabilities and to eliminate variants that are not usable or only usable with a lot of effort. Exemplarily, the approach with special touch gestures (as described in Section 0) did not seem promising and has therefore been prioritized lower. Contrariwise, other forms of input have shown positive aspects, like the application of physical pressure to a whole device instead of the need to point to some specific touch area, or the manipulation of magnetic fields with an specifically 


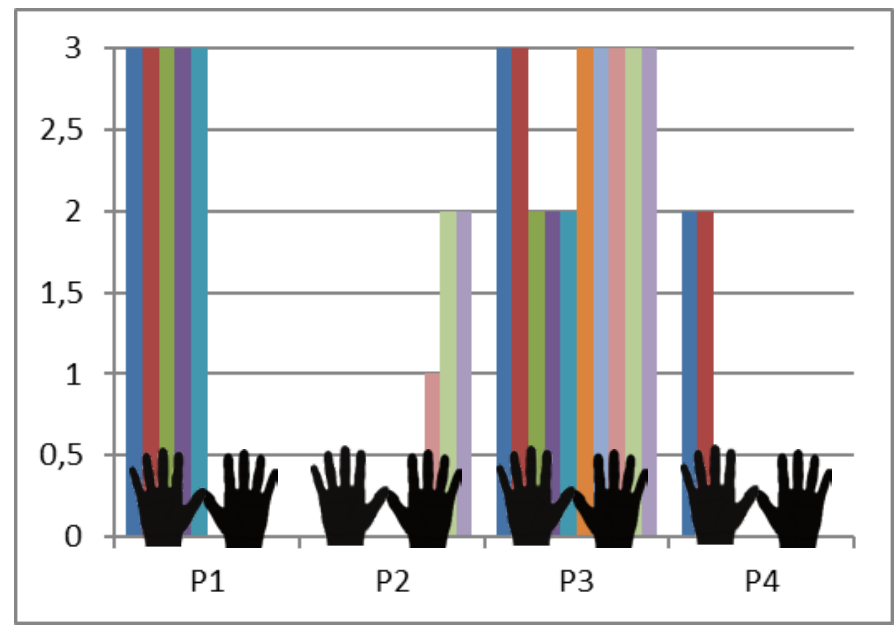

Fig. 13. Participants` abilities to extend single fingers. Please note that P1 could extend all fingers of the right hand simultaneously only.

constructed stylus pen with the exception that the interaction might have to be reduced to basic pointing and applying pressure instead of drawing (complex) shapes (as shown in Sections A and V.B).

Additionally, the observation helped to establish first parameters that could find their way into an early user model capable of storing data later needed to tailor the interaction to the specific user's needs and also to allow for suggestions regarding which input modalities a user is best capable of using. It also helped us to select in a first step appropriate

\section{REFERENCES}

[1] X. A. Chen, T. Grossman, D. J. Wigdor and G. Fitzmaurice, "Duet: Exploring Joint Interactions on a Smart Phone and a Smart Watch," in Proceedings of the SIGCHI Conference on Human Factors in Computing Systems (CHI '14), Toronto, Ontario, Canada, 2014, pp. 159-168.

[2] S. Hwang, A. Bianchi and K. Y. Wohn, "VibPress: Estimating Pressure Input Using Vibration Absorption on Mobile Devices," in Proceedings of the 15th International Conference on Human-computer Interaction with Mobile Devices and Services (MobileHCI '13), Munich, Germany, 2013, pp. 31-34.

[3] S. Hwang, A. Bianchi, M. Ahn and K. Y. Wohn, "MagPen: Magnetically Driven Pen Interactions on and Around Conventional Smartphones," in Proceedings of the 15th International Conference on Human-computer Interaction with Mobile Devices and Services (MobileHCI '13), Munich, Germany, 2013, pp. 412-415.

[4] S. Hwang and K. Y. Wohn, "PseudoButton: Enabling Pressure-sensitive Interaction by Repurposing Microphone on Mobile Device," in CHI '12 Extended Abstracts on Human Factors in Computing Systems (CHI EA '12), Austin, Texas, USA, 2012, pp. 1565-1570.

[5] M. Augstein and W. Kurschl, "Modelling Touchless Interaction for People with Special Needs," in Proceedings of the 20th Workshop on Adaptivity and User Modeling, Munich, Germany, 2014, pp. 157-166.

[6] W. Kurschl, M. Augstein, H. Stitz, P. Heumader and C. Pointner, "A User Modelling Wizard for People with Motor Impairments," in Proceedings of the 11th International Conference on Advances in Mobile Computing \& Multimedia (MoMM2013), Vienna, Austria, 2013.

[7] W. Kurschl, M. Augstein, T. Burger and C. Pointner, "User Modelling for People with Special Needs," in International Journal of Pervasive Computing and Communications (IJPCC), Vols. 10, No. 3, 2014.

[8] C. Harrison, R. Xiao, J. Schwarz and S. E. Hudson, "TouchTools: leveraging familiarity and skill with physical tools to augment touch interaction," in CHI '14 Proceedings of the SIGCHI Conference on Human Factors in Computing Systems, Toronto, Ontario, Canada, 2014 pp. 2913-2916. 